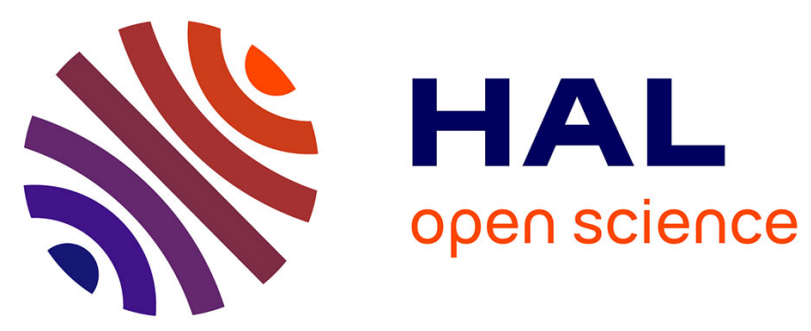

\title{
NeuroBetaMed: A re-configurable wavelet-based event detection circuit for in vitro biological signals
}

Adam Quotb, Yannick Bornat, Matthieu Raoux, Jochen Lang, Sylvie Renaud

\section{To cite this version:}

Adam Quotb, Yannick Bornat, Matthieu Raoux, Jochen Lang, Sylvie Renaud. NeuroBetaMed: A reconfigurable wavelet-based event detection circuit for in vitro biological signals. Circuits and Systems (ISCAS), 2012 IEEE International Symposium on, May 2012, Seoul, South Korea. pp.1532 - 1535. hal-00742324

\section{HAL Id: hal-00742324 \\ https://hal.science/hal-00742324}

Submitted on 16 Oct 2012

HAL is a multi-disciplinary open access archive for the deposit and dissemination of scientific research documents, whether they are published or not. The documents may come from teaching and research institutions in France or abroad, or from public or private research centers.
L'archive ouverte pluridisciplinaire HAL, est destinée au dépôt et à la diffusion de documents scientifiques de niveau recherche, publiés ou non, émanant des établissements d'enseignement et de recherche français ou étrangers, des laboratoires publics ou privés. 


\title{
NeuroBetaMed: A re-configurable wavelet-based event detection circuit for in vitro biological signals
}

\author{
Adam Quotb*,Yannick Bornat*, Matthieu Raoux ${ }^{\S}$, Jochen Lang ${ }^{\S}$ and Sylvie Renaud* \\ ${ }^{*}$ Univ. Bordeaux, IPB, IMS, UMR 5218, F-33400 Talence, France. \\ ${ }^{*}$ CNRS, IMS, UMR 5218, F-33400 Talence, France. \\ §Univ. Bordeaux, CBMN, UMR 5248, F-33600 Pessac, France.
}

\begin{abstract}
We present a reconfigurable acquisition and wavelet-based detection circuit, NeuroBetaMed, for in vitro biological signals. It is implemented on a configurable digital integrated circuit (FPGA). We consider real-time computing as a hard specification and silicon area as a price to pay. It is designed for noisy signals like those recorded from in vitro cellular preparations, by extracellular electrodes. NeuroBetaMed performs biological signal acquisition, stationary wavelet transform (SWT) and adaptive thresholding to detect action potentials (APs). Initially developed to detect pancreatic islet cells action potentials, this system is also suitable for neural signals.
\end{abstract}

\section{INTRODUCTION}

Analyzing and understanding electrical signals in human body is the ultimate goal of many scientists in the field of bioscience. This work proposes insights on analyzing tools for better decoding cortical or pancreatic islet cells signals. Deciphering communication between neurons and networks is for example mandatory to make prosthesis intended to overcome handicaps such as blindness or lesion in the spinal cord. The electrical activity of pancreatic islet cells relates nutrient (e.g. glucose) and hormone (e.g. incretins) blood concentrations to insulin secretion. Decoding the activity of these cells may be an approach to control insulin delivery in certain types of diabetes [10]. For such applications, realtime processing of bio-signals is required. The implementation proposed here fits hard real-time constraints, meaning that processing induced by an incoming sample is finished before the next sample arrives.

A classical initial step for biosignals decoding is the identification of action potentials (APs), as elements of the electrical influx exchange between cells (neurons or pancreatic islet cells). An AP is a pulse in the membrane voltage of an excitable biological cell after a membrane depolarization, in response to a stimulation.

Automatic biopotential detectors have been proposed for realtime detection ([2] and [3]). The most intuitive method to extract APs is to compare the raw signal with a threshold. This threshold is related to the signal amplitude and can adapt to changes in environmental conditions. This method, hereafter called raw thresholding, is only efficient when APs have significantly higher amplitude than noise. However for long term or large scale acquisitions that require extracellular electrodes (usually arranged in matrices), the low signal to noise ratio (SNR) makes raw thresholding inefficient. Alternatively a pre-processor may be used which amplifies AP shapes and attenuates out-of-band noise before signal comparison. Pre-processing methods for spikes detection have been described (Teager Energy Operator, Normalized Cumulative Energy Difference, Phase-Space, Summation, Convolutionbased template matching) [6] and [7]. However, these methods are not efficient for signals with an SNR below 5. This justifies investigations on alternative spike detection techniques dedicated to low SNR signals.

The wavelet transform was considered only recently as a relevant pre-processing method in the context of neural signal processing. It has been successfully used in biomedical applications [4] and [5]. This method is interesting due to its performance in terms of compression and time-frequency localization for feature extraction. Some wavelet-based methods were already successfully implemented for off-line neural processing [9]. Wavelet transform detection methods were tested only with simulated or pre-recorded neural signals, for specific spike shapes. Some were implemented in software with no real-time computation perspective.

We propose in this paper a new method based on wavelet transform that performs adaptive AP detection on in vitro neural and pancreatic islet cells signal in real-time. We present the architecture of the system implementing that method. We also illustrate our technique with experiments performed online with in vitro pancreatic islet cells. Moreover, we will detail its performance in terms of detection.

\section{WAVELET TRANSFORM ALGORITHM}

Our strategy for real-time AP detection is to implement a pre-processor which emphasizes APs shapes and attenuates out-of-band noise. The output of this pre-processor is compared to a threshold to discriminate AP events. The preprocessing algorithm is the Stationary Wavelet Transform (SWT). The detection system computes in real-time the SWT, the adaptive threshold and the comparison. We evaluated Stationary Wavelet Transform (SWT) to be the best compromise to optimize AP detection rate and time computation cost [11]. SWT is a time-frequency analysis, based on filter bank decomposition (fig.1) on an orthogonal basis, performed by the convolution of an input signal $x[n]$ and two filters $(\mathrm{G}$ and $\mathrm{H})$. These filters define the mother wavelet. Some parameters are closely related: the shape of the detected signal and the mother wavelet on one hand, the sampling frequency, the duration of the detected signal and the focused detail level on the other 


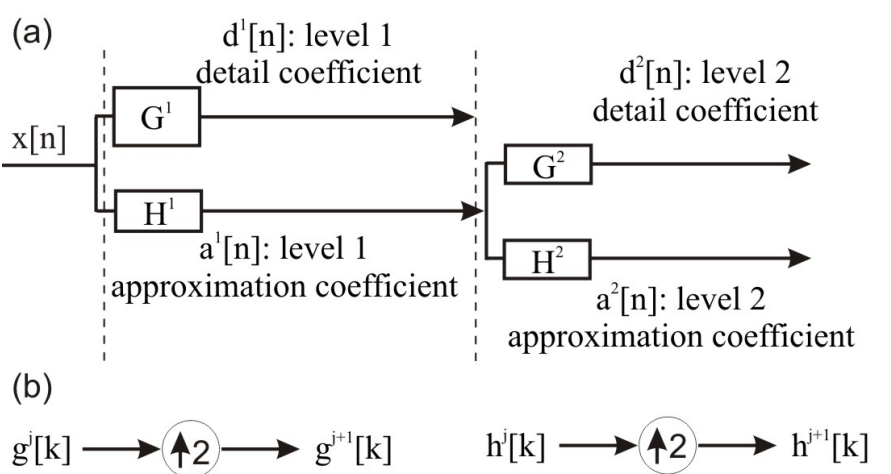

Fig. 1. a) Two-level SWT filter bank implementation. $x[n]$ : input signal, b) Low-pass $(\mathrm{H})$ and high-pass filters $(\mathrm{G})$ up-sampling coefficients. j: wavelet decomposition level.

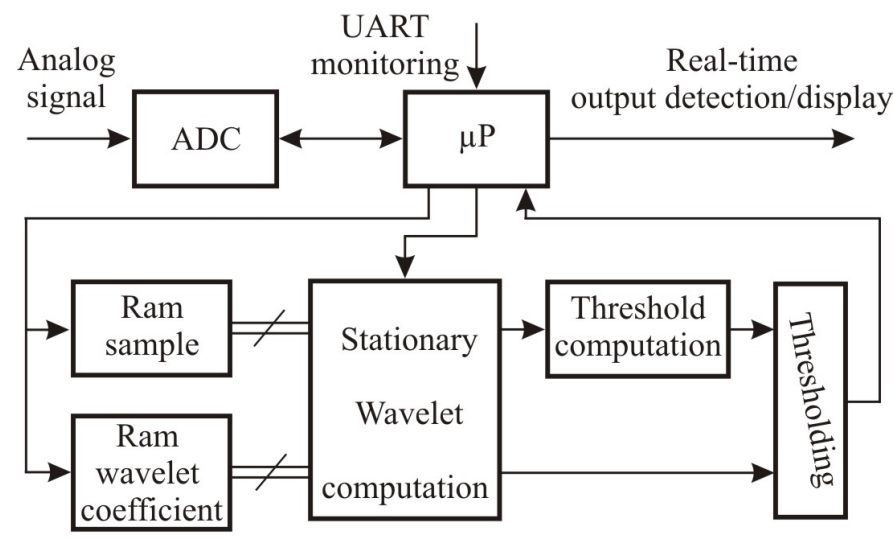

Fig. 2. FPGA block diagram.

hand. Concerning mother wavelets, we can find in the literature several examples particularly performing when dealing with APs (Haar, Daubechies, B-spline [1]).

\section{SYSTEM ARCHITECTURE}

We implemented a NeuroBetaMed prototype on a FPGA using the Xilinx Spartan-3A starter kit. It performs acquisition and wavelet-based AP detection on one input signal. Our goal is to validate real-time performance on a single channel before considering multi-channels processing adapted for example to recordings from multi-electrode arrays. Fig. 2 represents the device architecture. Data processing is controlled by a 8-bit RISC microprocessor $(\mu \mathrm{P})$. It controls computation modules (SWT computation, threshold computation), manages incoming samples from an analog-to-digital converter (ADC), and performs a UART interface to update the detection parameters and retrieve results. Detection outputs are both sent through FPGA I/Os for real-time display and read by the processor for statistical analysis.

\section{A. Biological signal acquisition block}

Mouse pancreatic islet cells were cultured on a commercial microelectrode array (MEA) from Multi Channel Systems (MCS). MEAs contain plates to record electrical signal and provide 60 analog read-out channels. The MCS acquisition set-up includes an integrated pre-amplifier with x1000 gain. After a basic signal conditioning due to the ADC (signal amplification with a variable gain and $1.25 \mathrm{~V}$ offset), we use the onboard FPGA 12bits ADC to sample the chosen channel at $10 \mathrm{kHz}(\mathrm{x}[\mathrm{n}])$. Once the $\mu \mathrm{P}$ retrieves a sample, it converts it into a 16 bits fixed point value and sends it to the SWT pre-processor. We use the direct implementation of SWT [1]. Detail and approximation outputs $d^{j}$ and $a^{j}$ from level j (fig.1) in direct SWT computation are given by equation 1 and 2 .

$$
\begin{aligned}
& d^{j}[n]=\sum_{k=0}^{7} g^{j}[k] \times a^{j-1}[n-k] \\
& a^{j}[n]=\sum_{k=0}^{7} h^{j}[k] \times a^{j-1}[n-k]
\end{aligned}
$$

where $\mathrm{j}$ represents the SWT level (up to 6), $\mathrm{k}$ the number of mother wavelet coefficient (up to 8) and $\mathrm{n}$ the sample number. $h^{j}$ and $g^{j}$ are low-pass and high-pass filter at level j. So a SWT computation for a sample at one level necessitates at most 16 multiplications and 14 additions. We use a pipelined architecture (fig.3) that only requires 1 multiplier and 1 adder. Samples and coefficients are stored in two different memories so two reading process are possible at the same time (at the first pipeline stage). The second pipeline stage performs the multiplication, and the last one performs the addition. To simplify the control state machine, we chose to compute 8 additions with a zeroed accumulator, instead of 7 with a random initial accumulator value. The result of the last addition is directly written in RAM. Each filter level requires 10 clock cycles. This represent $0.2 \mu \mathrm{s}$, as our FPGA is clocked at $50 \mathrm{MHz}$.To keep this time, we also restrained from computing unnecessary levels of details. In terms of computation time, between the arrival of a sample and the computation of the 6th wavelet detail level (maximum computation), our system requires $1.4 \mu \mathrm{s}$. Considering a $10 \mathrm{kHz}$ sampled signal, we can use a single SWT pre-processor to compute up to 70 channels using time multiplexing.

\section{B. Adaptive threshold detection block}

The SWT module provides the first level $d^{1}$ and the most effective detail level $\left(d^{k}\right)$ used for threshold application in AP detection. We dynamically estimate the standard deviation $(\sigma)$ of the first detail level $d^{1} ; d^{1}$ is computed from the raw input signal by applying a SWT high-pass filter. We use a feedback loop to perform $\sigma$ computation. This loop checks the ratio of samples above the current $\sigma$ and compares it to a reference value (P). When the loop is stabilized the estimation of $\sigma$ is correct [3]. Within the loop, the signal and its estimated $\sigma$ are compared by the comparator A. The output of A is a binary pulsatile signal, which mean value is a linear representation of the ratio of samples above the estimated $\sigma$. A low-pass filter (F1) extracts this mean value with a gain of G1. The difference between this value and a reference $\mathrm{P}$ is fed back to the comparator A. This signal is the estimation of the input data standard deviation $\sigma$. The user must set the reference $\mathrm{P}$ depending on the approximate distribution of the processed signal. For a white Gaussian noise signal, the probability of a sample absolute value to be over $\sigma$ is 0.159 . In that case the 


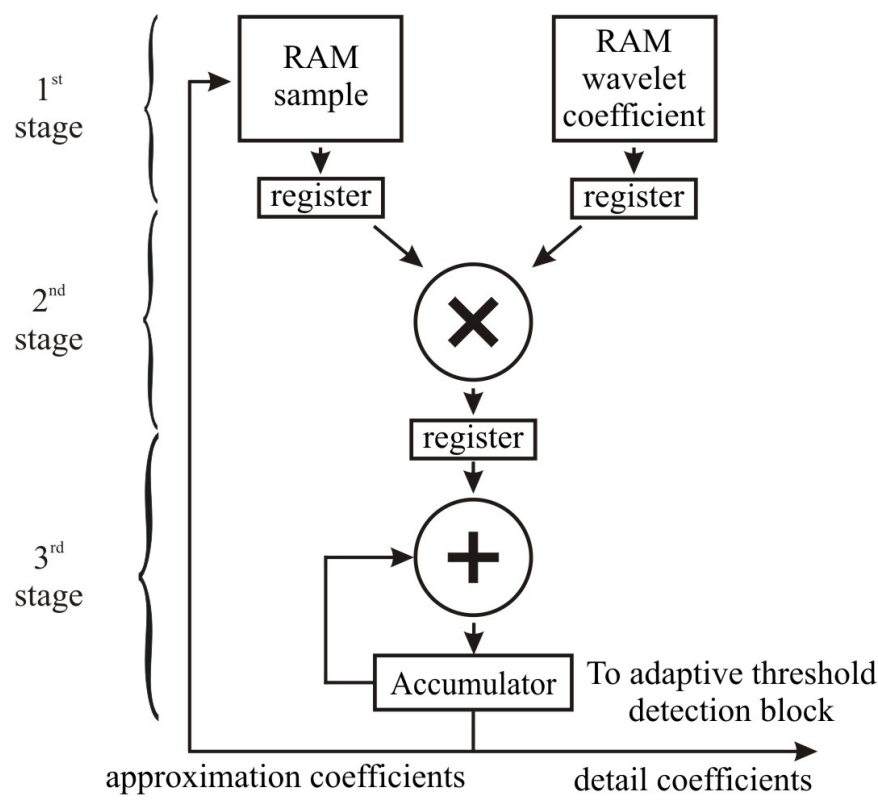

Fig. 3. SWT three stage pipelined architecture.

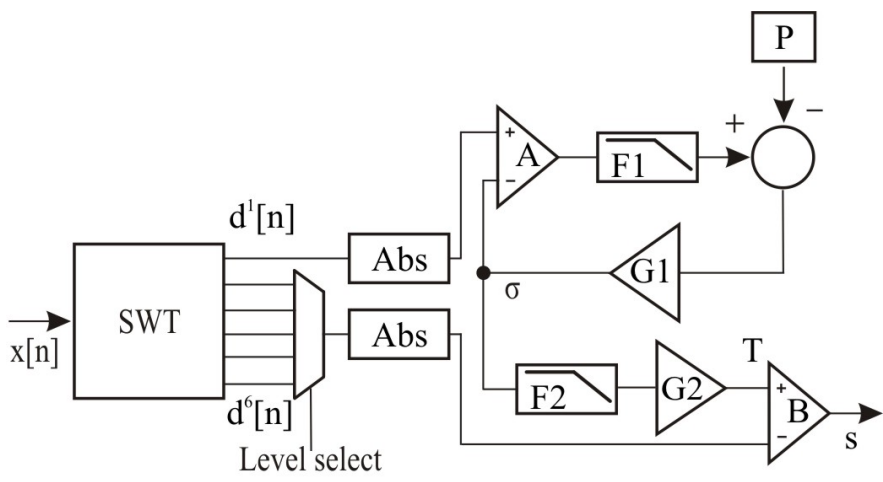

Fig. 4. Adaptive threshold detection module.

reference in the control loop is set to 0.159 . Finally, after a low-pass filter (F2) that stabilizes its value, the threshold $\mathrm{T}$ is calculated as shown in equation (3), where G2 gain is in the range of 0 and 3 .

$$
T=G 2 \times \sigma
$$

The transfer function of the digital low-pass filters F1 and F2 is:

$$
H(z)=K \times \frac{1-w_{z} z^{-1}}{1-w_{p} z^{-1}}
$$

$\mathrm{H}$ is a Butterworth low-pass filter which cut-off frequency is $10 \mathrm{~Hz}$ and sampling frequency is $10 \mathrm{kHz}$. Due to the hardware implementation, we encode $\mathrm{H}$ parameters in fixed-point 16 bits format. $\mathrm{K}=0.003, w_{z}=1$ and $w_{p}=-0.99$. Six wavelet detail level are at the disposition of the user in order to apply the threshold (levels 1 to 6). An AP is detected if the absolute value of the most efficient detail level chosen by the user is above $\mathrm{T}$. A block diagram of the entire wavelet detection module is presented in fig. 4 .
TABLE I

FPGA LOGIC UTILIZATION

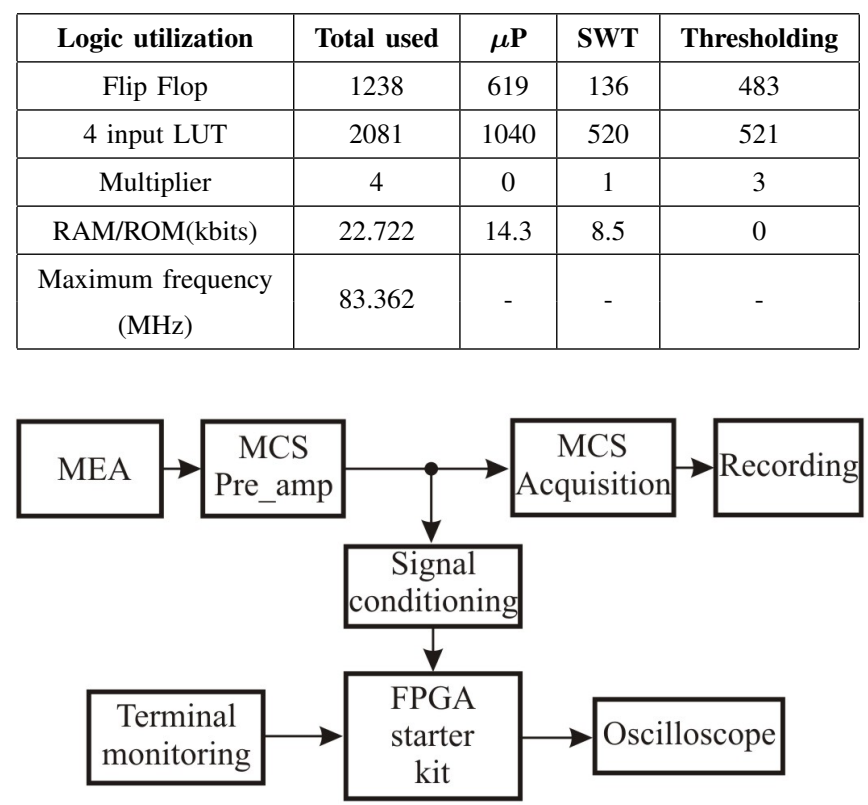

Fig. 5. Acquisition and AP detection experiment configuration.

\section{RESULTS}

\section{A. Implementation results}

We implemented the acquisition and detection system in a FPGA from Xilinx (Spartan-3A XC3S700A) using the ISE tool chain. The whole system is clocked at $50 \mathrm{MHz}$. To store the set of samples necessary for computing the 5 approximation levels, we use a $(512 \times 16)$ bits RAM block. We use two other RAMs $(8 \times 16)$ bits to store mother wavelet coefficients. In terms of device utilization, table I summarizes the FPGA post-synthesis area cost for NeuroBetaMed blocks. The critical resource in our application is the RAM, mostly used by the SWT pre-processor. Indeed, contrary to the $\mu \mathrm{P}$ which can be considered to have fixed cost, SWT RAM size is directly related to the number of processed channels: for $\mathrm{n}$ channels, the necessary SWT RAM size is $0.25+n * 8$ kbits. For signals that require a lower SWT detail level, RAM usage may be reduced to $0.25+n$ kbits. SWT RAM usage can also be reduced by using a lower sampling frequency. We did not investigate this yet, as hardware anti-aliasing filters in the MCS set-up cannot be reconfigured and consequently bound the sampling frequency.

\section{B. Acquisition results}

Our acquisition and wavelet detection system was used on in vitro recording of pancreatic islet cell culture. We used simultaneously a reference commercial acquisition system from MCS, as represented in fig.5. The MCS software acquisition software is commonly used by biologists for AP detection. In fig.6, we plot the variations of the adaptive threshold signal, for a MEA signal with a high SNR (SNR $\geq 5)$, recorded on 
pancreatic islets. The FPGA system requires $30 \mathrm{~ms}$ to set the threshold due to the time constant present in filter F1 (see fig.4). After $30 \mathrm{~ms}$ the threshold can be considered to follow equ.3. In fig.7.A, a recorded raw pancreatic islet cells signal is given. Note that field potentials were recorded and signals have downward deflection. 9 spikes above $+/-30 \mathrm{mV}$ were identified and marked by arrows. In order to evaluate our circuit's performance in terms of AP detection at low $\mathrm{SNR}$, we added to the original signal a gaussian noise as explained in [8] and obtain pancreatic islet cells signal with added noise (fig.7.B). This signal is then applied in real time to our circuit by a signal wave generator (Agilent 33250A), so the detection is made from the signal shown in figure (fig.7.B). The sixth wavelet detail level and the detection circuit outputs are represented (fig.7.C and D). The detection output shows that, out of 9 APs, 7 were correctly detected and 1 false positive (circle in fig.7.D) is present with our circuit although visual detection of these APs in fig.7.B is almost impossible. This example, is typical of good detection scores we obtain on equivalent MEA signals, and shows the good AP detection rate of our circuit in low SNR conditions.

\section{CONCLUSION}

We present in this paper a new integrated acquisition and detection system, NeuroBetaMed, based on Stationary Wavelet Transform. It provides embedded on-line processing of in vitro signals. Good performance in AP detection were obtained on pancreatic islet cells cultures which SNR is particularly low. Thanks to its re-configurability, this system can be easily ported to other types of biological signals, like neural signals, with a low computational cost. NeuroBetaMed will be soon tested on ex vitro preparations of spinal cords. We presented a FPGA implementation flow and experimental results for a single processing channel; this concept will be extended to 60 channels processing. We expect that system to provide novel integrated processing solutions for embedded Brain Machine Interfaces that require optimized integration density and realtime data analysis.

\section{ACKNOWLEDGMENT}

We thank Gilles N'Kaoua for technical support. This work was supported by the ANR program HYBIOPACS (ANR2010-EMMA-031-01).

\section{REFERENCES}

[1] I. Daubechies. Ten lectures on wavelets. Philadelphia: Society for Industrial and Applied Mathematics, 1992.

[2] B. Gosselin and M. Sawan. An ultra low-power cmos automatic action potential detector. IEEE Transactions on Neural Systems and Rehabilitation Engineering, 17:346-353, 2009.

[3] R. R. Harrison. A low-power integrated circuit for adaptive detection of action potentials in noisy signals. In Engineering in Medicine and Biology Society, volume 4, pages 3325-3328, Seattle, 2003.

[4] K. Imfeld, A. Maccione, M. Gandolfo, S. Martinoia, P.-A. Farine, M. Koudelka-Hep, and L. Berdondini. Real-time signal processing for high-density microelectrode array systems. International Journal of Adaptive Control and Signal Processing, 23:1099-1115, 2009.

[5] J. Lee and D. Kipke. Neural signal processing using discrete wavelet transform for neural interfaces. In International Conference on Microtechnologies in Medicine and Biology, pages 169-172, 2006. start

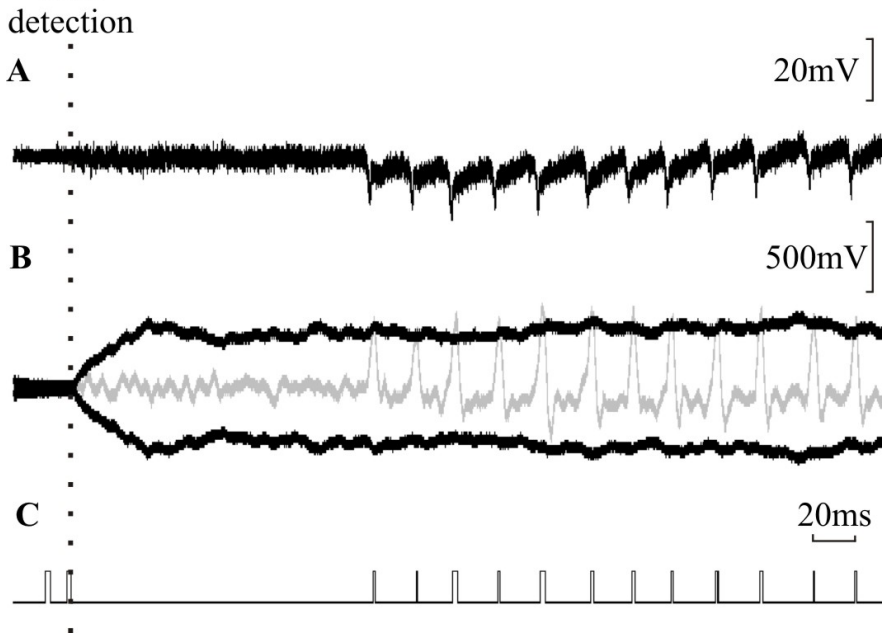

Fig. 6. NeuroBetaMed outputs: (A) raw signal from pancreatic islet cells with high SNR, (B) sixth wavelet detail $d^{6}$ (grey) and adpative thresholds (black) and (C) detection output.

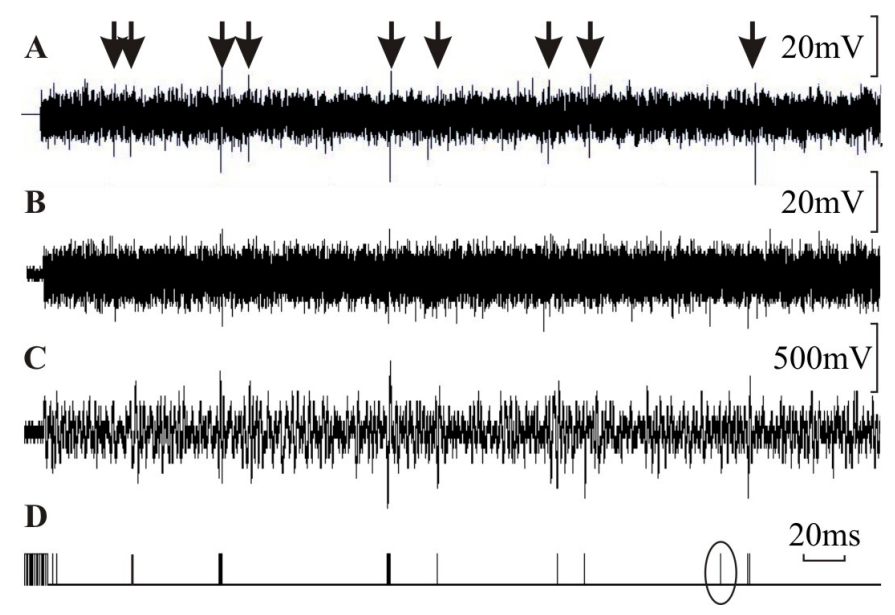

Fig. 7. NeuroBetaMed outputs: (A)raw signal from pancreatic islet cells. Note that field potentials were recorded and spikes have a downward reflection. Signals above $+/-30 \mathrm{mV}$ were retained as spike and are indicated by arrows above the trac, (B) pancreatic islet cells signal with added noise (low SNR) from which the detection is applied, (C) sixth wavelet detail $d^{6}$ and (D) detection output.

[6] N. Mtetwa and Leslie S. Smith. Smoothing and thresholding in neuronal spike detection. Neurocomputing, 69:1366-1370, 2006.

[7] C. Paterson, R. Curry, A. Purvis, and S. Johnson. Detection of action potentials in the presence of noise using phase-space techniques. In World Academy OF Sience, Engineering and Technology, volume 34,7779, 2008.

[8] A. Quotb, Y. Bornat, and S. Renaud. Wavelet transform for realtime detection of action potentials in neural signals. Frontiers in Neuroengineering, 4, 2011.

[9] A. Salmanpour, Lyndon J. Brown, and J. Kevin Shoemaker. Performance analysis of stationary and discrete wavelet transform for action potential detection from sympathetic nerve recordings in humans. In International Conference on Engineering in Medicine and Biology Society, pages 2932-2935, 2008.

[10] S. Seino and I. (Eds.) Bell. Pancreatic Beta Cell in Health and Disease. Springer Verlag Tokyo, 2008.

[11] Y. Yuning, A. Kamboh, and J. M. Andrew. Adaptive threshold spike detection using stationary wavelet transform for neural recording implants. Biomedical Circuits and Systems Conference, pages 9-12, 2010. 